\title{
Gestures Recognition Method Based on Electromyographic Signal
}

\author{
Tian Yucheng ${ }^{a}$ Wang Mo $^{b}$ Zhang Xing $^{c}$ Wang Xin'an ${ }^{d}$ \\ Peking University Shenzhen Graduate School, Shenzhen 518055, China \\ a452765021@qq.com b 913856544@qq.comc zhx@pku.edu.cn \\ d wangxa@pkusz.edu.cn
}

Keywords EMGs; Power frequency interference; Wavelet transform; Neural networks

\begin{abstract}
Different gestures were identified through analyzing and processing the electromyographic signal(EMGs) collected from the forearm. That in turn was used to control the upper limb rehabilitation equipment. The wavelet denoising was used after filtering the power frequency interference and the normalized processing. The high and low frequency coefficients were decomposed from signal through wavelet transform. The variance calculated from the frequency coefficients was used as a characteristic value. Through the neural networks classification, the recognition rates of seven kinds of gestures are over 99\%. The seven kinds of gestures were wrist inward, wrist outward, fist stretch, fist clench, wrist up, wrist down and palm downward spiral.
\end{abstract}

\section{Introduction}

Stroke or spinal cord injury often cause movement disorders such as hemiplegia. The current rehabilitation training on such patients relies mainly on one-to-one manual operation between the physician and the patient. The treatment effect relies heavily on physician level, and it's ineffective. As a kind of intelligent rehabilitation equipment ${ }^{[1,2]}$, exoskeleton rehabilitation system can provide the patient with auxiliary force or guide patients in rehabilitation exercise according to predetermined trajectory.

EMGs is a physiological signal acquired from the electrical activity of subcutaneous muscle. The basis of the exoskeleton rehabilitation system controlled by EMGs is the EMGs pattern recognition. For the EMGs pattern recognition, Pingao Mei et al. put forward the method that uses the time-frequency features as the input of Elman neural network, in order to recognize four kinds of gestures with the average recognition rate of $92.5 \%{ }^{[3]}$. Yi-Hung Liu et al. adopted the improved support vector machine (SVM) to recognize some kinds of gesture with the correct rate $93.54 \%{ }^{[4]}$. Kosmidou et al. used two-channel electrical sensors to recognize 9 kinds of American sign language with the recognition rate of more than $97 \%$.

The process of EMGs processing in this paper is shown in figure 1, including the steps such as collection, preprocessing, feature extraction, pattern recognition and so on. The wavelet denoising was used to pretreat the data after filtering $50 \mathrm{~Hz}$ power frequency interference and harmonic. Then, the feature parameters of different gestures were extracted through the wavelet transform. The parameters then were put into the neural network. The accurate identification of a variety of gestures was realized after training and recognizing.

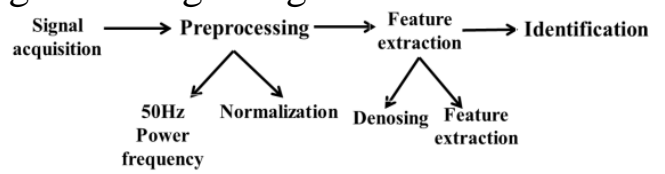

Fig. 1EMGs pattern recognition process

\section{Acquisition and Preprocessing of EMGs}

\section{EMGs Acquisition}

All the data used in this paper were collected from the surface of the forearm muscles. The seven kinds of gestures were wrist inward, wrist outward, fist stretch, fist clench, wrist up, wrist down, 
palm downward spiral and so on. Electrode position and the seven kinds of gestures were shown in figure 2. Sampling frequency was $1 \mathrm{KHz}$, every action did 20 groups and each group did 3 times. The duration of each group was at least 4s. Had rested at least $1 \mathrm{~min}$ after 20 groups, in order to eliminate the impact of the muscle fatigue. Four-channel hand EMGs were collected and the action misjudgment rate had been lowered preliminarily due to the characteristics of the coordination between four muscles.

\section{Narrow-bandwidth Butterworth Band-stop Filter}

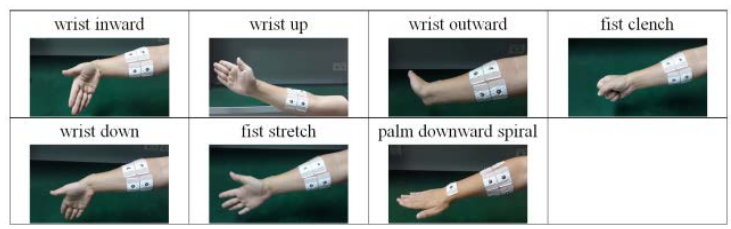

Fig. 2 Gestures and electrode position

The identification of the useful signal would be affected by the $50 \mathrm{~Hz}$ frequency interference included in the signal. Narrow-bandwidth butterworth band-stop filter ${ }^{[5]}$ was adopted to remove the interference of $50 \mathrm{~Hz}$ and its harmonics. The value of the frequency response of the ideal filter was 0 , while its frequency range which was to be eliminated was $\left[\omega_{1}, \omega_{2}\right]$. The value was 1 in other frequency. The frequency response formulas in the ideal situation were shown as follows.

$$
\left|H\left(e^{j \omega}\right)\right|=\left\{\begin{array}{c}
0 \omega_{1}<\omega<\omega_{2} \\
1 \text { else }
\end{array}\right.
$$

The power frequency interferences of $50 \mathrm{~Hz}, 100 \mathrm{~Hz}$ and $150 \mathrm{~Hz}$ were filtered through the use of the narrow-bandwidth butterworth band-stop filter. The stop bands were $49 \sim 51 \mathrm{~Hz}, 99 \sim 101 \mathrm{~Hz}$ and 149 151 Hz, respectively. The spectrums between and after filtering the power frequency interference of $50 \mathrm{~Hz}$ was shown in figure 3. The figure showed that it was decreased obviously at $50 \mathrm{~Hz}$ after filtering.

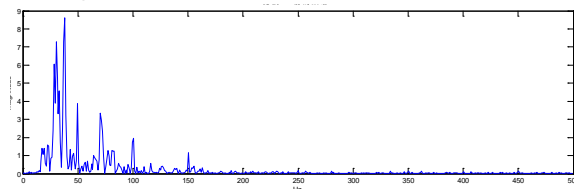

(a) Beforefiltering

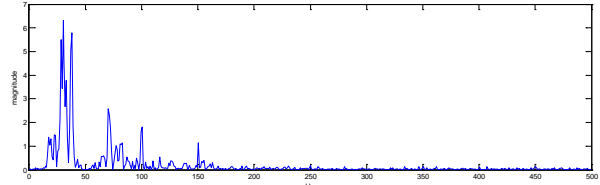

(b) After filtering

Fig. 3 The spectrum before and after filtering $50 \mathrm{~Hz}$ power frequency

The signal figures between and after filtering were shown in figure 4. Take the first group of the action wrist inward as an example, the identification of useful signal was affected by the power frequency, especially the judgment of the action starting moment, shown in figure 4. After filtering, the signal amplitude in the period without action decreased significantly which made the contrast between the period with action and without action more significant. Also the differences between different movements were enlarged, which was useful for the action identification and provided guarantee for the high identification rate.

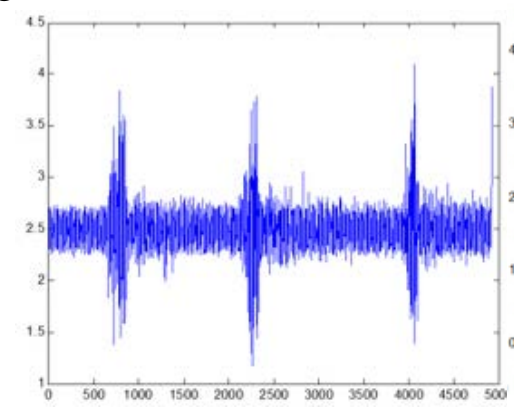

(a) Beforefiltering

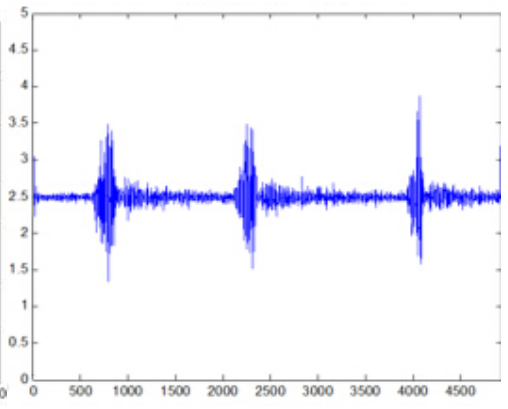

(b) After filtering

Fig. 4 The signal figures before and after filtering power frequency 


\section{Wavelet Denoising and Feature Extraction}

The widely popular time-frequency analysis method in the area of EMGs processing is the wavelet transform $^{[6]}$. The wavelet transform has a great localization property in the time as well as the frequency domain and the zoom microscopic features in the high frequency component. Its role is similar to the bandpass filter with a set of equal bandwidth and variable center frequency. This features before are particularly suited to process EMGs which is mutant. The method of multiresolution wavelet transform was used to process the EMGs, in order to extract the variances of the wavelet coefficients under different scales as the eigenvalues.

\section{Wavelet Denoising}

The signals are decomposed into high and low frequency parts after layering through wavelet transform and denoising ${ }^{[7]}$. Wavelet denoising changes the digital quantity in the high frequency part to get rid of the digital mixed with noise because the white noise is usually appear in the high frequency part. The purpose of denoising is to restrain the noise part and reduce the real signal from the data.

Sym5 wavelet was adopted to decompose the signal into 3 layer in this paper; The threshold of wavelet denoising was automatically generated by using the wavelet threshold function ddencmp; According to the threshold of each layer, soft threshold method was used for signal denoising. The so-called soft threshold was that the data was set to 0 if the absolute value was less than the given threshold, others shrunk to zero. The EMGs figures of the action wrist inward before and after wavelet denoising were shown in figure 5. As shown in the figure, the contrast between the useful and the useless signal was more significant. And the signal amplitude, relative to the baseline, in the period without action almost became zero. Also, the differences between different movements were further enlarged, which provided guarantee for the high identification rate.

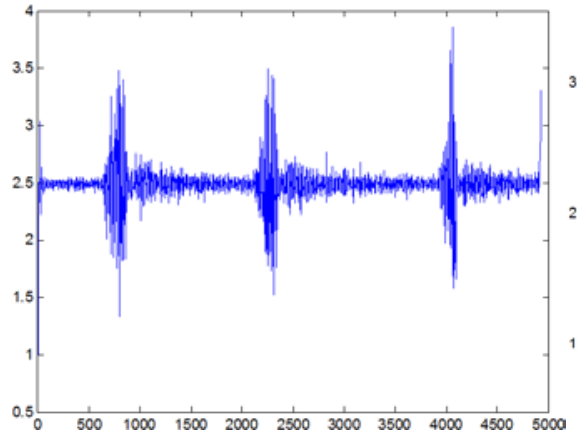

(a) Before denoising

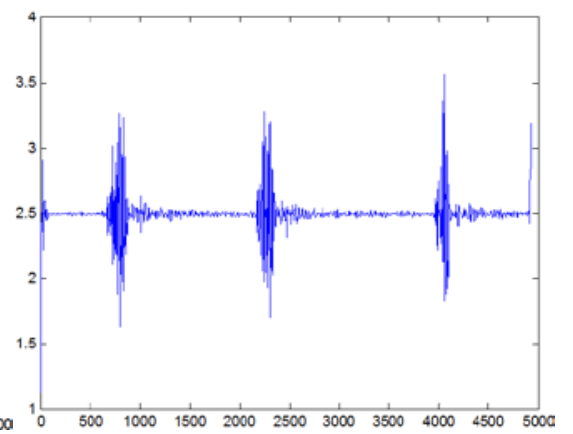

(b) After denosing

\section{Features extraction}

Fig. 5 The signal figures before and after wavelet denoising

The low-frequency part was decomposed step by step while the high-frequency part was ignored in the multi-resolution analysis of wavelet transform. A 3-level wavelet decomposition structure was shown in figure 6 . As is shown, a relatively high frequency part $W_{i}$ anda relatively low frequency part $V_{i}$ were decomposed from the low-frequency part of the upper level. And then, only the lower part went on to decompose, generating the higher part $W_{i-1}$ and the lower part $V_{i-1}$. The original signal $V_{3}$ can be obtained through the lowest frequency $V_{0}$ superimposed with the higher frequency in each layer, that is $V_{3}=V_{0}+W_{0}+W_{1}+W_{2}$.

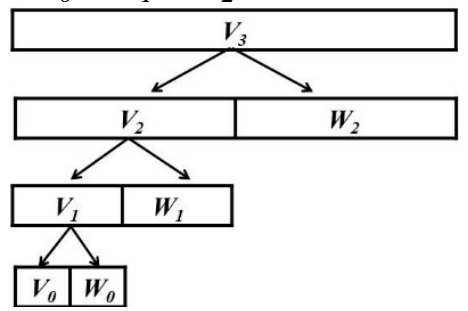

Fig. 6 Three-level wavelet decomposition tree

The variances of the wavelet coefficients under different scales were extracted as the eigenvalues after 3-level decomposition. For every channel, the number of eigenvalue was four, that was the lowest frequency part and the higher frequency part in each level. The four-channel eigenvalues of 
the first experiment of action wrist inward after the feature vector normalization processing were shown in table 1 . As is shown, the lower frequency part contributes more than $89 \%$, which is consistent with the fact that the variance of action part is large and the signal is largely concentrated in the low frequency part. Therefore, the eigenvalue of the lowest frequency part was the only value used. At last, a four-dimension feature vector was obtained, such as [0.98486, 0.89035, 0.9358, 0.9557]. The burden of neural network was effectively reduced under the premise that did not affect the classification accuracy. And the classification speed was improved.

Table 1 The feature vector of the first experiment of action wrist inward

\begin{tabular}{|l|c|c|c|c|}
\hline & Lowest frequency & Low frequency & High frequency & Highest frequency \\
\hline Channel 1 & 0.98486 & 0.0022567 & 0.005996 & 0.0068863 \\
\hline Channel 2 & 0.89035 & 0.029668 & 0.044746 & 0.035238 \\
\hline Channel 3 & 0.9358 & 0.016979 & 0.027892 & 0.019333 \\
\hline Channel 4 & 0.9557 & 0.045381 & 0.02103 & 0.018727 \\
\hline
\end{tabular}

\section{Classification and Identification}

\section{Feedforward Neural Network}

Neural network is a network composed of a large number of neurons connected with each other. Neural network consists of feedforward neural networks ${ }^{[8]}$, feedback neural network and self-organizing neural network according to the way it connects. The feedforward neural networks was used in this paper.

The inputs of neurons in feedforward neural network were from the previous layer and output to the next layer after being dealt with the activation function. As shown in figure 7(a), $x_{i}$ is the input and $f$ is the activation function. For the input nodes, they just need to output to the next layer after being dealt with the activation function. Different from the input nodes, computing nodes, in addition to the input nodes, have more than one input and only one output. Feedforward network generally divides into layers and the input is just linked with the output of the previous layer. And just the input and the output nodes are linked to the outside. The layers in the middle are called hidden layer. The network structure is shown in figure 7(b), three layers , in turn, are input, hidden and output. It is 2-layer network as the input unit is not neuron.

The feedback appears only in the process of training rather than test process. The data can only spread forward until the output layer in the test process without backward feedback. The improved BP neural network, belongs to the feedforward neural network, was adopted in this paper.

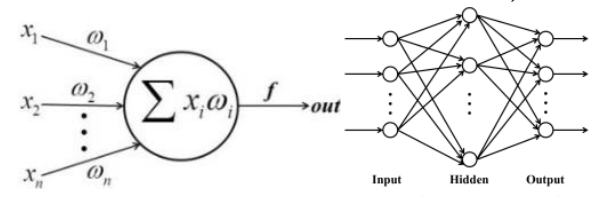

(a) Neuron

(b) Neural network structure

Fig. 7 Neuron and neural network structure in the neural network

\section{Classification and Identification}

The input node number in the neural network was 4 corresponding to the dimension of the eigenvector. The S-type function was adopted as the activation function of the node, that is $f(x)=\frac{1}{1+e^{-x}}$. Levenberg-Marquardt algorithm ${ }^{[9]}$, LM for short, was used as the learning algorithm. Compared with BP algorithm, LM had less time of iterations, faster convergence speed and high precision.

The seven kinds of gestures which were wrist inward, wrist outward, fist stretch, fist clench, wrist up, wrist down and palm downward spiral were numbered respectively from 1 to 7 on behalf of the categories. The target vector of the classifier was expressed by $O U T=\left[O_{1}, \ldots \ldots, O_{7}\right]$ and only one element was equal to 1 while others were 0 . The position of the element which was equal to 1 corresponded to the corresponding action. For example, the target vector of the action fist stretch, 
whose category number was 2, was $O U T=\left[\begin{array}{lllllll}0 & 1 & 0 & 0 & 0 & 0 & 0\end{array}\right]$. In the actual test classification, the value of a node was made 1 if the value of this output node was the maximum, while other values became 0 .

Amounted to 140 sets of data which belonged to the seven kinds of action. Five sets of each action were extracted as the test data and the other 105 sets as training data. The identification results were shown in table 2.

As is shown in table 2, the accurate identification rate changed little with the same actions which were to be identified and different number of nodes in the hidden layer. Thus, the accurate identification rate will not be affected by the number of nodes in the hidden layer. However, the greater the number of nodes, the bigger the network and the longer the running time of the program. Therefore, selected the number of hidden nodes as small as possible under the premise of ensuring relatively high recognition rate. As is shown in the last column of table 2, the recognition rate maintained over $99 \%$ no matter what the group of actions were. Similarly, as shown in last row, the recognition rate maintained over $99.5 \%$ while the actions to be recognized were different and the number of nodes in the hidden layer was the same.

Table 2 The recognition results

\begin{tabular}{cccccccccc}
\hline Category/Hidden & 8 & 10 & 15 & 20 & 25 & 30 & 35 & 40 & Mean \\
\hline$a b c$ & 100 & 100 & 100 & 100 & 100 & 100 & 99.94 & 100 & 99.993 \\
$e f g$ & 100 & 100 & 99.3 & 100 & 99.3 & 99.3 & 95.71 & 98.57 & 98.883 \\
abcd & 100 & 100 & 100 & 100 & 99.5 & 100 & 100 & 99.5 & 99.875 \\
defg & 99.47 & 100 & 99.47 & 100 & 100 & 99.47 & 100 & 100 & 99.8 \\
bcde & 100 & 100 & 100 & 100 & 100 & 100 & 100 & 100 & 100 \\
aceg & 99.47 & 100 & 99.47 & 98.47 & 99.15 & 98.95 & 98.42 & 99.47 & 99.175 \\
abcde & 100 & 100 & 100 & 100 & 100 & 100 & 100 & 100 & 100 \\
bcdef & 100 & 100 & 100 & 99.58 & 100 & 100 & 99.58 & 100 & 99.895 \\
cdefg & 99.58 & 100 & 99.58 & 100 & 100 & 100 & 100 & 100 & 99.895 \\
abcdef & 98.62 & 99.66 & 99.66 & 100 & 100 & 100 & 100 & 100 & 99.743 \\
bcdefg & 99.31 & 99.31 & 99.66 & 99.66 & 99.31 & 100 & 100 & 100 & 99.656 \\
abdefg & 99.66 & 99.66 & 99.66 & 100 & 100 & 100 & 100 & 99.66 & 99.83 \\
abcdefg & 99.7 & 99.2 & 100 & 99.7 & 98.82 & 100 & 100 & 99.7 & 99.64 \\
Mean & 99.678 & 99.833 & 99.754 & 99.784 & 99.698 & 99.825 & 99.51 & 99.75 &
\end{tabular}

Notes: $a, b, c, d, e, f, g$ represent seven kinds of action, $a b c$ represents the actions to be recognized, abcdefg means seven actions to be recognized; Get the corresponding recognition rates while the number of nodes in the hidden layers changes. The numbers changed among 8 、 10、15、20、25、30、35、40; The marks of mean in the last column and row correspond to the average recognition rates of a column and a row respectively; The random number was used when initializing the network. Therefore, the network structure would be some different in different time even if the data used was the same. That, in turn, led to the deviation of the recognition rate before and after. In order to reduce the impact of this situation, 10 times a group were tested and then the average of that 10 times was computed.

\section{Result and Analysis}

Three aspects which were preprocessing, feature extraction and classification of the EMGs were studied in this paper. Four-channel hand EMGs were collected and the action misjudgment rate had been lowered preliminarily due to the characteristics of the coordination between four muscles. Narrow-bandwidth butterworth band-stop filter was adopted to remove the interference of $50 \mathrm{~Hz}$ and its harmonics which made the contrast between the period with action and without action more significant and enlarged the differences between different movements, which was useful for the action identification and provided guarantee for the high identification rate. After the wavelet denosing, the differences between different movements were further enlarged and the signal 
amplitude in the period without action became almost zero. One eigenvalue in each channel was extracted from wavelet coefficients. For different actions, four eigenvalues were different with each other, that was, the excitement degrees of four muscles were different which accorded with reality. In the stage of identification, BP neural network was adopted while the LM algorithm was used as the learning algorithm. The recognition rate was over 99\% regardless of what the action and the number of nodes in the hidden layer. Recognition effect is significant.

\section{Acknowledgments}

This paper is supported by the basic research project of Shenzhen science and technology project, and the project fund number is JCYJ20140710093817639. Also, thanks for my teacher Wang Xin'an, and thanks for his supportment.

\section{References}

[1].Johnson W B, Fatone S, Gard S A. Walking mechanics of persons who use reciprocating gait orthoses[J]. Rehabilitation Research and Development, 2009, 46(3): 435-446.

[2].Lo A C, Guarino P D, Richards L G, et al. Robot-assisted therapy for long-term upper-limb impairment after stroker[J]. New England Journal of Medicine, 2010, 362(19): 1772-1783.

[3]. Mei P G, Luo Z Z. sEMG disposal based on wavelet packet analysis and Elman neural network[J]. Mechanical \& Electrical Engineering Magazine. 2008, 25(1): 7-10.

[4].Liu Y H, Huang H P, Weng C H. Recognition of Electromyographic Signals Using Cascaded Kernel Learning Machine[C]. IEEE/ASME Transactions on Mechatronics, 2007, 12(3): 253-264.

[5].Zhang W X. The Design of Butterworth Digital Notch Filter[J]. Modern Electronic Technology, 2008(18): $1-3$.

[6]. Englehart K, Hudgins B, Parker P A. A wavelet-based continuous classification scheme for multifunction myoelectric control[J], IEEE Transactions on Biomedical Engineering, 2001, 48 (3): 302-311.

[7]. Yan R. The Application of BP Neural Network Based on Wavelet Denoising in Prediction of Deformation[D]. The master's degree thesis of the geologic university of China (Beijing), 2012.

[8].Jiang M H, Gielen G, Zhang B, et al. Fast Learning Algorithms for Feedforward Neural Networks[J]. Applied Intelligence, 2003, 18(1): 37-54.

[9].Zhao H, Zhou R X, Lin T Q. Neural Network Supervision and Control Based on Levenberg-Marquardt Algorithm [J]. Journal of Xi'an Jiao Tong University, 2002, 36(5): 2-3. 